\title{
nad $A$ and nadB of Shigella flexneri $5 a$ are antivirulence loci responsible for the synthesis of quinolinate, a small molecule inhibitor of Shigella pathogenicity
}

\author{
Anne-Laure Prunier, ${ }^{1}$ Raymond Schuch, ${ }^{1} \dagger$ Reinaldo E. Fernández, ${ }^{1}$ \\ Karen L. Mumy, ${ }^{2,3}$ Henrik Kohler, ${ }^{2,3} \ddagger$ Beth A. McCormick ${ }^{2,3}$ \\ and Anthony T. Maurelli ${ }^{1}$
}

Correspondence

Anthony T. Maurelli

amaurelli@usuhs.mil

Received 12 February 2007

Revised 4 April 2007

Accepted 5 April 2007

\author{
${ }^{1}$ Department of Microbiology and Immunology, Uniformed Services University of the Health \\ Sciences, F. Edward Hébert School of Medicine, Bethesda, MD 20814-4799, USA \\ ${ }^{2}$ Mucosal Immunology Laboratory, Department of Pediatric Gastroenterology and Nutrition, \\ Massachusetts General Hospital, Charlestown, MA 02129, USA \\ ${ }^{3}$ Department of Microbiology and Molecular Genetics, Harvard Medical School, Boston, \\ MA 02115, USA
}

\begin{abstract}
The evolution of bacterial pathogens from commensal organisms involves virulence gene acquisition followed by pathoadaptation to the new host, including inactivation of antivirulence loci (AVL). AVL are core ancestral genes whose expression is incompatible with the pathogenic lifestyle. Previous studies identified cadA (encoding lysine decarboxylase) as an AVL of Shigella spp. In this study, AVL of Shigella were identified by examining a phenotypic difference from its non-pathogenic ancestor, Escherichia coli. Unlike most E. coli strains, Shigella spp. are nicotinic acid auxotrophs, the pathway for the de novo synthesis of NAD being uniformly defective. In Shigella flexneri, this defect is due to alterations in the nad $A$ and/or nadB genes encoding the enzyme complex that converts L-aspartate to quinolinate, a precursor to NAD synthesis.

Quinolinate was found to inhibit invasion and cell-to-cell spread of Sh. flexneri $5 a$ and its ability to induce polymorphonuclear neutrophil transepithelial migration. Virulence of other Shigella species was also inhibited by quinolinate. Introduction of functional nad $A$ and nad $B$ genes from $E$. coli $\mathrm{K}-$ 12 into Sh. flexneri 5 a restored its ability to synthesize quinolinate but also resulted in strong attenuation of virulence in this strain. The results define nadA and nadB as AVL in Shigella and validate the concept of pathoadaptive evolution of bacteria from commensal ancestors by inactivation of AVL. They also suggest that studies focusing on this form of bacterial evolution can identify novel inhibitors of virulence in other bacterial pathogens.
\end{abstract}

\section{INTRODUCTION}

The evolution of bacterial pathogens from their commensal ancestors is believed to occur by the acquisition of genes that allow the organism to colonize a new ecological niche. A 'quantum leap' in the evolution of a bacterium occurs

tPresent address: Laboratory of Bacterial Pathogenesis and Immunology, The Rockefeller University, 1230 York Avenue, NY 10021, USA.

‡Present address: Universitätsklinikum Erlangen, Fredrich-AlexanderUniversität, Loschgestr. 15, 91054 Erlangen, Germany.

Abbreviations: $A V L$, antivirulence loci; $C R$, Congo red; EIEC, enteroinvasive E. coli; EPEC, enteropathogenic E. coli; fMLP, $N$-formylmethionyl leucyl phenylalanine; PMN, polymorphonuclear neutrophil; QUIN, quinolinate; TSB, tryptic soy broth; T3SS, type III secretion system. when new genes are acquired en bloc via horizontal transfer of plasmids or bacteriophages (Groisman \& Ochman, 1996). The presence of virulence genes in 'pathogenicity islands' reflects this form of gene acquisition (Lee, 1996). Thus, horizontal gene transfer can radically transform an organism from a benign resident of a eukaryotic host into a pathogen capable of colonizing a new niche within the host and, incidentally, causing harm to the host.

A complement to the process of bacterial pathogens' evolution by gene acquisition is the progressive adaptation of the bacteria to the new niche by mutation and selection for improved fitness. This process is called pathoadaptive evolution (Sokurenko et al., 1999). An important element of this process is the selection of 'black holes' in pathogen genomes, that is, the inactivation or loss of genes that are 
incompatible with, and even antagonistic to, the new pathogenic lifestyle (Maurelli, 2007). These incompatible genes, which we define as antivirulence loci (AVL), are present in the genome of the non-pathogenic ancestor but absent or inactive in the pathogen because expression of AVL in the pathogen is detrimental to expression of some virulence phenotype. Thus, deletion or mutational inactivation of AVL is selected for in the pathogen.

Bacteria of the genus Shigella are the causative agents of bacillary dysentery in humans and primates. In the early stage of the disease, Shigella produce several enterotoxins that are believed to facilitate their passage from the small intestine to the colon (Fasano et al., 1997). Once in the colon, the bacteria invade colonic epithelial cells, replicate intracellularly, and spread to adjacent cells. Intracellular bacterial growth and tissue destruction lead to an intense inflammatory response with migration of polymorphonuclear neutrophils (PMNs) to the site of infection (Sansonetti et al., 1999).

We previously demonstrated the contribution of 'black holes' to the evolution of Shigella from the non-pathogenic gut commensal Escherichia coli (Maurelli et al., 1998). The cadA gene, encoding lysine decarboxylase in E. coli K-12, was shown to be part of a large region uniformly altered in all Shigella spp., and its expression is incompatible with several phenotypes of Shigella virulence (Day et al., 2001; McCormick et al., 1999). These observations support the concept of bacterial pathogen evolution by selection for increased fitness through gene inactivation or deletion.
The genetic similarities between Shigella and E. coli are sufficient to justify grouping them in the same genus. Shigella spp. may even be considered clones, or subgroups, of E. coli (Pupo et al., 2000). However, there are several wellknown metabolic differences that distinguish the organisms and aid in their clinical differentiation. Among these are auxotrophic requirements of Shigella that are not found among isolates of E. coli. Most clinical isolates of Shigella fail to grow on minimal medium. Of these auxotrophic isolates, $98 \%$ grow on minimal medium supplemented with methionine, tryptophan and nicotinic acid (Ahmed et al., 1988). Many strains of Shigella require only nicotinic acid supplementation for growth on minimal medium. This requirement is due to mutations in $n a d A$ and/or $n a d B$ in $S h$. flexneri (Gemski et al., 1971; Mantis \& Sansonetti, 1996)

The L-aspartate-dihydroxyacetone phosphate pathway is used by E. coli K-12 for de novo synthesis of NAD (Fig. 1). LAspartate oxidase, encoded by $n a d B$, forms a multi-enzyme complex with quinolinate synthetase $\mathrm{A}$, the product of nadA. This enzyme complex catalyses the oxidation of Laspartate to iminoaspartate, which is then condensed with dihydroxyacetone phosphate (DHAP) to form quinolinate (QUIN). Quinolinate phosphoribosyltransferase, the product of nadC, converts QUIN to nicotinic acid mononucleotide, which enters the pathway for NAD synthesis. In the absence of functional nadA and/or nadB, QUIN is not made and no nicotinic acid mononucleotide is available to synthesize NAD. However, exogenous nicotinic acid can be converted to nicotinic acid mononucleotide by the action of nicotinate phosphoribosyltransferase, the product of $p n c B$

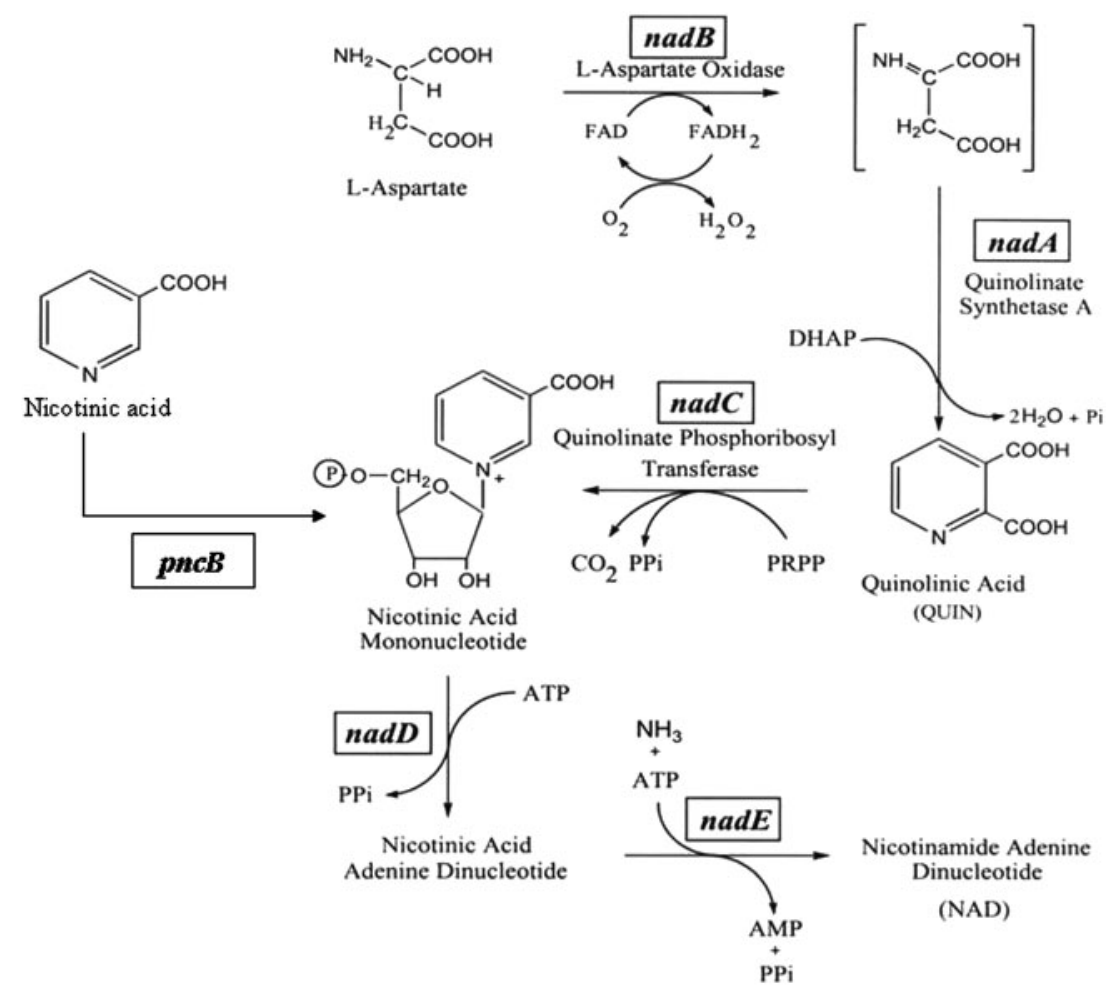

Fig. 1. L-Aspartate-dihydroxyacetone phosphate pathway for de novo NAD synthesis in E. coli K-12 [adapted from Flachmann et al. (1988)]. 
(Fig. 1). This pathway bypasses the need for nadA and nadB and synthesis of QUIN and is the basis of the nicotinic acid requirement for Shigella growth on minimal medium (Gemski et al., 1971).

The following study determined whether the auxotrophic requirement for nicotinic acid in Sh. flexneri represents another example of pathoadaptation through selection for loss of a gene function that is incompatible with virulence. We hypothesized that selection for pathoadaptation has led to inactivation of nadA and/or nadB in Shigella and enteroinvasive E. coli (EIEC). Our results demonstrate that $n a d A$ and $n a d B$ are AVL and that QUIN, an intermediate in the biosynthesis of NAD, is a potent small molecule inhibitor of several virulence phenotypes of Shigella.

\section{METHODS}

Bacterial strains and media. Strain M90T is the wild-type strain of Sh. flexneri 5a (Sansonetti et al., 1982) used throughout this study. Other strains of Shigella used were: 2457T (Sh. flexneri 2a) (Formal et al., 1958) and its avirulent, plasmid-cured derivative strain BS103 (Maurelli et al., 1984); BS504 (Sh. dysenteriae 1), BS512 (Sh. boydii), and BS514 (Sh. sonnei). BS176 is a spontaneous virulence-plasmidcured derivative of M90T (Sansonetti et al., 1986). Overnight cultures for virulence assays were inoculated from single pigmented colonies growing on tryptic soy broth (TSB, Bacto) plates with $1.5 \%$ agar and $0.025 \%$ Congo red (CR; Sigma) at $37{ }^{\circ} \mathrm{C}$ (Maurelli et al., 1984) and grown in Luria-Bertani (LB) medium at $37{ }^{\circ} \mathrm{C}$. The wild-type strain of Salmonella typhimurium employed was $\chi 3306$ (Gulig \& Curtiss, 1987). ATM266 is an EIEC strain (Harris et al., 1982). E2348/69 is a strain of enteropathogenic E. coli (EPEC) obtained from Gail Hecht (University of Illinois at Chicago, USA). E. coli K-12 MC4100 was the source of DNA for PCR reactions to amplify the nadA and nadB loci. Ye37 is a Yersinia enterocolitica isolate WA obtained from Peter Feng (FDA, College Park, MD, USA). BS813 is the $\operatorname{nadA}^{+}$nadB transformant of M90T.

For plating efficiency determination of M90T, M9 glucose minimal medium supplemented with methionine $\left(10 \mu \mathrm{g} \mathrm{ml}^{-1}\right)$ and tryptophan $\left(20 \mu \mathrm{g} \mathrm{ml}^{-1}\right)$ was used. When required, $1 \mathrm{mM}$ QUIN (Fluka), or $10 \mu \mathrm{g} \mathrm{ml}^{-1}$ nicotinic acid (Sigma) was added.

Molecular techniques. The primers used for amplification of the $n a d A$ and $n a d B$ loci were as follows: nadAPF $5^{\prime}$-CAAGCAACTCTATGTCGGTGG-3' and nadAM 5'-TGGCAAGGCCAATACACAGC-3' allowed amplification of the entire nadA locus and its promoter, while nadBPF 5 '-CAAAGGGTTAGAGTGTCTCG-3' and nadBM 5'-CGTGGGCCAGACCAGAACTATTCC-3' allowed amplification of the entire nadB locus and its promoter, using Taq DNA polymerase (Qiagen).

For cloning, we used the commercially available plasmids pGEM-T (Promega) and pBluescript II-SK (Stratagene). Transformants were obtained by electroporation of competent cells from cold water treatment and screened for ampicillin resistance. Ampicillin-resistant clones were then confirmed by PCR and their phenotype was monitored by determining plating efficiency on minimal medium containing the appropriate supplements.

Invasion assays. Invasion assays were performed as described previously (Sandlin et al., 1996) with some alterations. Bacteria were grown overnight at $37^{\circ} \mathrm{C}$ then diluted $1: 50$ into $5.0 \mathrm{ml} \mathrm{LB}$ and grown with agitation at $37^{\circ} \mathrm{C}$ to $\mathrm{OD}_{600} \sim 0.6$. Standardized culture volumes were removed, diluted, titered, and used to infect semi- confluent monolayers of mammalian cells at $37{ }^{\circ} \mathrm{C}$ in Dulbecco's modified essential medium (DMEM) with or without the indicated concentrations of QUIN, nicotinic acid or nicotinic acid mononucleotide (Sigma). The input inoculum was $\sim 1 \times 10^{8}$ c.f.u. in a volume of $1 \mathrm{ml}$. After $30 \mathrm{~min}$, infected monolayers were washed extensively with $1 \times$ PBS to remove both the QUIN and extracellular bacteria and incubated for an additional $90 \mathrm{~min}$ in DMEM with $50 \mu \mathrm{g}$ gentamicin $\mathrm{ml}^{-1}$. Monolayers were subsequently washed with PBS and lysed in the presence of $0.5 \%$ Triton X-100. Released intracellular bacteria were plated on TSB-CR agar and colonies were counted to determine the percentage invasion compared to the input titre. The same protocol was used to test the effect of QUIN on intracellular growth of bacteria except that the lysis step was performed after $0.5,1.5,2.5,3.5,4.5$ and $5.5 \mathrm{~h}$.

Alternatively, instead of lysing the infected cells, monolayers were fixed with methanol and stained with Giemsa 45, 90, 135 or $180 \mathrm{~min}$ after final gentamicin treatment, and examined by light microscopy for evidence of bacterial invasion. At least 200 infected cells were counted. The frequency of protrusion formation, indicating intracellular spreading capacity of the bacteria, was determined as the percentage of cells with internalized bacteria that displayed cytoplasmic protrusions.

Plaque assay. Plaque assays were performed essentially as described previously (Oaks et al., 1985) with some alterations. Bacterial cultures were grown overnight, diluted 1:50 into $5.0 \mathrm{ml}$ fresh LB, and grown with agitation at $37^{\circ} \mathrm{C}$ to $\mathrm{OD}_{600} \sim 0.6$. Standardized culture volumes were removed, diluted, titered, and used to infect confluent monolayers of HeLa cells for $2 \mathrm{~h}$ at $37{ }^{\circ} \mathrm{C}$ in a $6.0 \% \mathrm{CO}_{2}$ atmosphere. The input inoculum was $\sim 1 \times 10^{4}$ c.f.u. in a volume of $100 \mu \mathrm{l}$. Agarose overlays with or without the indicated concentrations of QUIN were then applied to infected monolayers and allowed to incubate at $37{ }^{\circ} \mathrm{C}$ in a $6 \% \mathrm{CO}_{2}$ atmosphere for an additional $72 \mathrm{~h}$. Neutral red staining allowed for enumeration of plaques to determine the plaque-forming efficiency compared to the input titre. Plaque diameter was calculated as the average of 10 plaques.

PMN transepithelial migration assay. PMN migration was assessed as previously described (McCormick et al., 1998). For assessment of Sh. flexneri-induced PMN migration in the presence of QUIN, $25 \mu \mathrm{l}$ washed bacterial suspension $\left(6 \times 10^{8}\right.$ bacteria $)$ was added directly to the basolateral surface (upper compartment) of inverted T84 cell monolayers for $90 \mathrm{~min}$ at $37{ }^{\circ} \mathrm{C}$. Next, the T84 cell monolayers were washed to remove non-adherent bacteria and QUIN ( 5 or $100 \mu \mathrm{M}$ ) was added to both the apical and basolateral reservoirs for $30 \mathrm{~min}$. PMNs isolated from volunteers were subsequently added to the basolateral surface $\left(1 \times 10^{6}\right.$ per monolayer $)$ and assessed for PMN transmigration in the continued presence of QUIN. Transmigration was quantified by assaying for myeloperoxidase, the PMN azurophilic marker, and is expressed as cell equivalents as described previously (McCormick et al., 1998; Parkos et al., 1991). A $1 \mu \mathrm{M}$ solution of $N$-formylmethionyl leucyl phenylalanine (fMLP; Sigma), a potent PMN chemoattractant, was used as a control.

Protein extraction and preparation. To study the amount of expressed proteins, $\sim 20 \mathrm{ml}$ of exponential cultures of M90T grown with or without $0.4 \mathrm{mM}$ QUIN were standardized using $\mathrm{OD}_{600}$ measurement and harvested by centrifugation $(13000 \mathrm{~g}, 5 \mathrm{~min})$. Supernatants were filtered through a $0.45 \mu \mathrm{m}$ pore size filter (Millipore) and secreted proteins were precipitated by the addition of trichloroacetic acid ( $10 \%$ final concentration), followed by acetone washing. The final pellet of precipitated proteins was resuspended in $200 \mu \mathrm{l}$ sample buffer. Whole-cell lysates were generated by washing the pellets once with $20 \mathrm{ml}$ ice-cold, sterile $1 \times \mathrm{PBS}$, followed by centrifugation of $1 \mathrm{ml}$ aliquots and suspension in $500 \mu \mathrm{l} \mathrm{Laemmli}$ sample buffer (Bio-Rad). 
To study the effect of QUIN on CR-induced secretion via the type III secretion system (T3SS), whole-cell fractions and supernatants of CRtreated Sh. flexneri were prepared as described previously (Bahrani et al., 1997). Briefly, $40 \mathrm{ml}$ samples from exponential cultures of M90T were harvested by centrifugation $(13000 \mathrm{~g}, 5 \mathrm{~min})$. Each pellet was resuspended in $2 \mathrm{ml}$ sterile $\mathrm{PBS}$ and split into two $1 \mathrm{ml}$ aliquots incubated with $30 \mu \mathrm{g} \mathrm{CR} \mathrm{ml}{ }^{-1}$ for $1 \mathrm{~h}$ at $37^{\circ} \mathrm{C}$, with or without $0.4 \mathrm{mM}$ QUIN. After the incubation period, the samples were centrifuged again (13000 g, $5 \mathrm{~min}$ ). Whole-cell lysates and precipitated proteins from the supernatants were prepared as described above.

To study the effect of QUIN on the low-calcium-induced T3SS of $Y$. enterocolitica we used the same protocol with the exception that CR was replaced by $5 \mathrm{mM}$ EGTA to chelate cations from the medium.

SDS-PAGE and immunoblotting. All samples were boiled and $10 \mu \mathrm{l}$ of each were loaded on $10 \%$ acrylamide SDS-PAGE gels as described by Laemmli (1970). After electrophoresis, proteins were transferred to a nitrocellulose membrane. Immunoblotting procedures were carried out with mouse monoclonal antibodies $2 \mathrm{~F} 1$ and 2G2, which recognize IpaB and IpaC, respectively (Mills et al., 1988). Horseradish peroxidase-labelled sheep anti-mouse IgG (Amersham Bioscience) was used as secondary antibody and visualized by enhanced chemiluminescence with the visualizer Western blot detection kit (Upstate) in a LAS-3000 system using Image reader LAS 3000 v2.1 software (Fuji.). The images obtained were analysed using Image Gauge v4.22 software (Fuji) allowing quantification of the signals observed. For the study of the effect of QUIN on the calcium-deprivation-induced T3SS of $Y$. enterocolitica, SDS-PAGE gels were stained with Imperial protein stain (Pierce).

\section{RESULTS}

\section{QUIN inhibits Shigella invasion of HeLa cells}

If an intermediate in the de novo pathway of NAD synthesis can attenuate Shigella pathogenesis, the selective pressure for pathoadaptation to inactivate one or more of the genes encoding enzymes in the pathway could explain the presence of mutations in nadA and/or nadB in Shigella spp. and EIEC. To test the hypothesis that nadA and/or $n a d B$ are AVL whose expression leads to the synthesis of an inhibitor of Shigella virulence, the ability of M90T to invade HeLa cells was assayed in the presence and absence of several intermediates in the NAD biosynthetic pathway.

Table 1. Effect of NAD biosynthetic pathway intermediates on the invasive phenotype of Sh. flexneri $5 a$

\begin{tabular}{|lc|}
\hline Treatment $^{\star}$ & Invasion (\%) $\dagger$ \\
\hline None & $0.11 \pm 0.023$ \\
$1 \mathrm{mM}$ nicotinic acid & $0.125 \pm 0.015$ \\
$1 \mathrm{mM}$ nicotinic acid mononucleotide & $0.1 \pm 0.019$ \\
$0.5 \mathrm{mM}$ QUIN & $0.0024 \pm 0.0004$ \\
\hline
\end{tabular}

*The compounds were present in the invasion assay only during the 30 min invasion period.

$\dagger$ Percentage of bacteria recovered from infected wells after gentamicin treatment relative to the number of bacteria used for invasion. Values shown are mean \pm SD from at least two independent experiments.
As shown in Table 1, $0.5 \mathrm{mM}$ QUIN almost completely inhibited invasion of HeLa cells by M90T, whereas $1 \mathrm{mM}$ nicotinic acid or nicotinic acid mononucleotide had no adverse effect on invasion. Addition of $1 \mathrm{mM}$ QUIN caused no visible changes in HeLa cell morphology and up to $2.5 \mathrm{mM}$ QUIN caused no change in $\mathrm{pH}$ of the tissue culture medium.

We next measured the inhibitory effect of QUIN over a range of concentrations on Shigella invasion into HeLa cells. These experiments showed that QUIN inhibited Shigella invasion in a dose-dependent fashion and that the presence of as little as $400 \mu \mathrm{M}$ QUIN almost completely inhibited invasion (Fig. 2).

\section{QUIN inhibits intercellular spread of Shigella}

Another hallmark of Shigella pathogenesis is its ability to spread from cell to cell within a confluent monolayer after the initial invasion step. A prerequisite for intercellular spread is the intracellular motility mediated by the ability of internalized Shigella to polymerize actin monomers in the cytoplasm (Bernardini et al., 1989). The formation of cytoplasmic protrusions extending from infected cells and containing one or more bacteria at the end is a reliable measure of intracellular motility. To examine the effect of QUIN on protrusion formation in Shigella-infected cells, bacteria were first allowed to invade semi-confluent HeLa monolayers and then $400 \mu \mathrm{M}$ QUIN was added and the monolayers incubated at $37{ }^{\circ} \mathrm{C}$. At various time points, the monolayers were fixed, stained and examined for protrusions emanating from infected cells. Fig. 3(a) shows that QUIN-treated monolayers displayed the same frequency of protrusion formation from Shigella-infected cells as untreated monolayers. Thus, QUIN does not inhibit the actin-based intracellular motility of Shigella.

The ability to spread from cell to cell within a confluent monolayer is a virulence phenotype measured by the plaque assay (Oaks et al., 1985). Since QUIN blocked

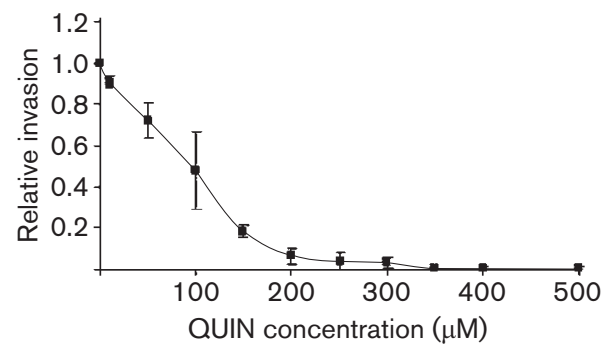

Fig. 2. Effect of QUIN on invasion of HeLa cells by M90T measured by the gentamicin protection assay. Percentage invasion was calculated as described in Methods. The results represent the mean $\pm S D$ of data obtained from two to four independent experiments for each concentration of QUIN, relative to the results obtained for M90T without QUIN (i.e. 1.0). 


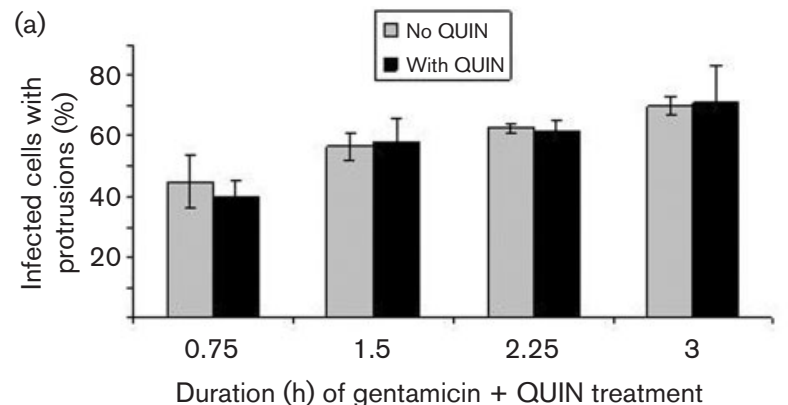

(c)

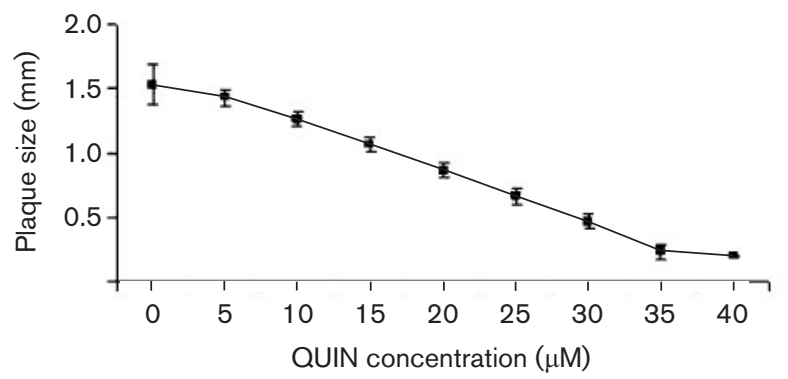

(b)

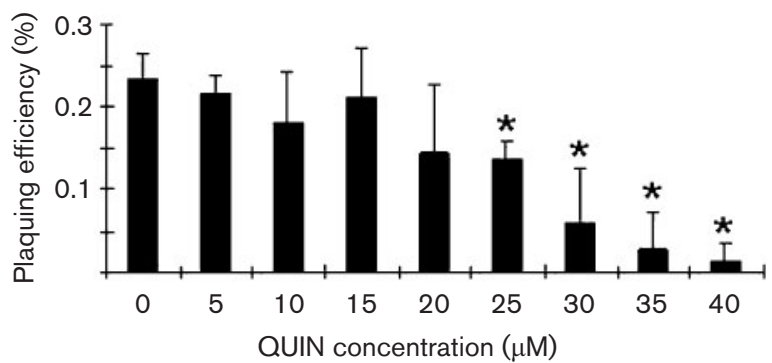

(d)

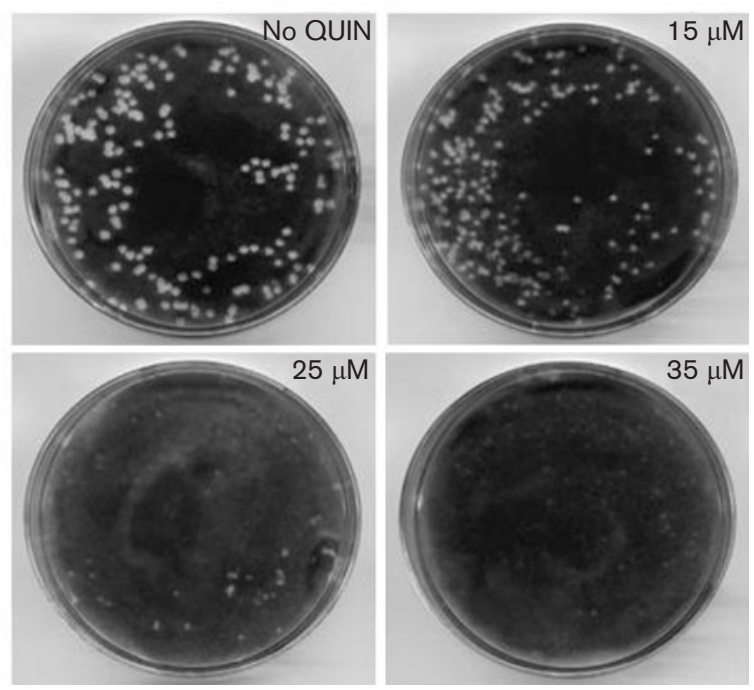

Fig. 3. Effect of QUIN on intra- and intercellular spreading by M9OT in HeLa cell monolayers. See Methods for details. (a) Effect of QUIN on protrusion formation. The results represent the mean \pm SD of data obtained from two independent experiments. (b) Effect of QUIN on plaque-forming efficiency. The results represent the mean \pm SD of data obtained from three independent experiments for each concentration of QUIN ( $\left.{ }^{*}, P<0.05\right)$. (c, d) Effect of QUIN on plaque size: (c) shows the mean \pm SD of plaque size measurements from the three experiments recorded in (b); (d) shows representative pictures of the plaques obtained for the control without QUIN as well as for three different QUIN concentrations (indicated in the upper right corner of each picture).

Shigella invasion it was added to infected monolayers only after an initial period in order to allow the bacteria to invade. Fig. 3(b) shows that QUIN impaired plaque formation in a dose-dependent manner and at much lower concentrations than were required to block invasion. The mean plaque diameter formed by M90T in the cell monolayer in the absence of QUIN was $1.53 \mathrm{~mm}$. Fig. 3(c) shows that the mean plaque size decreased with increasing concentrations of QUIN. This effect of QUIN on plaque size was accompanied by a marked effect on plaque shape, with QUIN causing the formation of faint and irregularly shaped plaques compared to the large, welldefined plaques of the control (Fig. 3d). Therefore, the major post-invasion effect of QUIN is a strong inhibition of the ability of the bacteria to spread from cell to cell, although it had no effect on the intracellular motility of Shigella.

\section{QUIN inhibits Shigella-induced transepithelial migration of PMNs}

A model system for the ability of Shigella to induce the severe inflammatory response typical of dysentery is the induction of PMN transepithelial migration across a monolayer of polarized T84 cells (McCormick et al., 1998). We tested QUIN for its effect on the ability of Shigella to induce transepithelial migration of PMNs after invasion of a monolayer of polarized epithelial cells. Since QUIN inhibited invasion of M90T into polarized T84 cells (Table 2), the assay was modified to allow the bacteria to invade the polarized T84 cells for $90 \mathrm{~min}$ before different concentrations of QUIN were added to the monolayers. Fig. 4 demonstrates that QUIN has a potent inhibitory effect on the ability of Shigella to induce transepithelial migration of PMNs. 
Table 2. Effect of QUIN on virulence phenotypes of Sh. flexneri, Sal. typhimurium and EPEC

\begin{tabular}{|c|c|c|c|}
\hline Strain & Treatment & $\begin{array}{c}\text { Invasion } \\
(\%)\end{array}$ & $\begin{array}{c}\text { PMN } \\
\text { migration* }\end{array}$ \\
\hline Sh. flexneri M90T & Buffer alone & 0.044 & $8.15 \times 10^{3} \dagger$ \\
\hline Sh. flexneri M90T & $100 \mu \mathrm{M}$ QUIN & 0.012 & $0.28 \times 10^{3} \dagger$ \\
\hline $\begin{array}{l}\text { Sal. typhimurium } \\
\text { x3306 }\end{array}$ & Buffer alone & 0.123 & $39.24 \times 10^{4}$ \\
\hline $\begin{array}{l}\text { Sal. typhimurium } \\
\text { x3306 }\end{array}$ & $100 \mu \mathrm{M}$ QUIN & 0.118 & $45.53 \times 10^{4}$ \\
\hline EPEC E2348/69 & Buffer alone & ND $\ddagger$ & $4.2 \times 10^{4}$ \\
\hline EPEC E2348/69 & $100 \mu \mathrm{M}$ QUIN & ND $\ddagger$ & $4.8 \times 10^{4}$ \\
\hline
\end{tabular}

${ }^{\star} \mathrm{PMN}$ migration was quantified as described in Methods.

$\dagger$ Results from Fig. 4.

$\ddagger \mathrm{ND}$, not done. EPEC strains are non invasive but still induce PMN migration.

Since QUIN was present in the medium throughout the experiment even after the PMNs were added, we tested the possibility that inhibition was due to a general effect of QUIN on PMN mobility. Control experiments showed that up to $100 \mu \mathrm{M}$ QUIN had no significant effect on PMN transepithelial migration in response to fMLP $\left(61.9 \pm 6.1 \times 10^{4}\right.$ cell equivalents in the absence of QUIN vs $57.3 \pm 11.4 \times 10^{4}$ and $51 \pm 4.6 \times 10^{4}$ cell equivalents with 5 and $100 \mu \mathrm{M}$ QUIN, respectively).

\section{QUIN is a specific inhibitor of Shigella virulence}

We tested another Sh. flexneri serotype as well as representative strains of Sh. dysenteriae, Sh. boydii, Sh. sonnei and EIEC to determine whether our observations on the inhibition of virulence by QUIN could be extended to

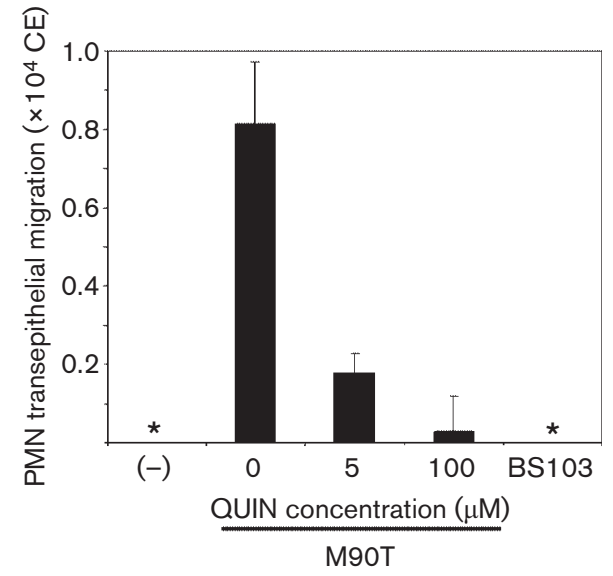

Fig. 4. Effect of QUIN on the ability of M90T to induce transepithelial migration of PMNs across T84 monolayers. See Methods for details. The three middle columns show PMN transmigration responses induced by wild-type Sh. flexneri $5 a$ M90T. BS103, a virulence-plasmid-cured Sh. flexneri strain, served as a negative control. The second negative control (-) represents PMN transmigration to buffer in the absence of bacteria or chemotactic stimulus. $\left(^{\star}\right)$ Both control PMN values were below the detection limit of the assay (700 PMNs). Data are expressed as mean $\pm S D$ for three monolayers in a single experiment and are representative of three separate experiments $(P<0.05)$. CE, cell equivalents.

the four species of Shigella as well as to the closely related EIEC. As shown in Fig. 5, $400 \mu \mathrm{M}$ QUIN largely inhibited invasion of mammalian cells by all these strains.

We next examined whether the inhibitory properties of QUIN on the virulence phenotypes of Shigella spp. could be extended to related invasive pathogens. Sal. typhimur-
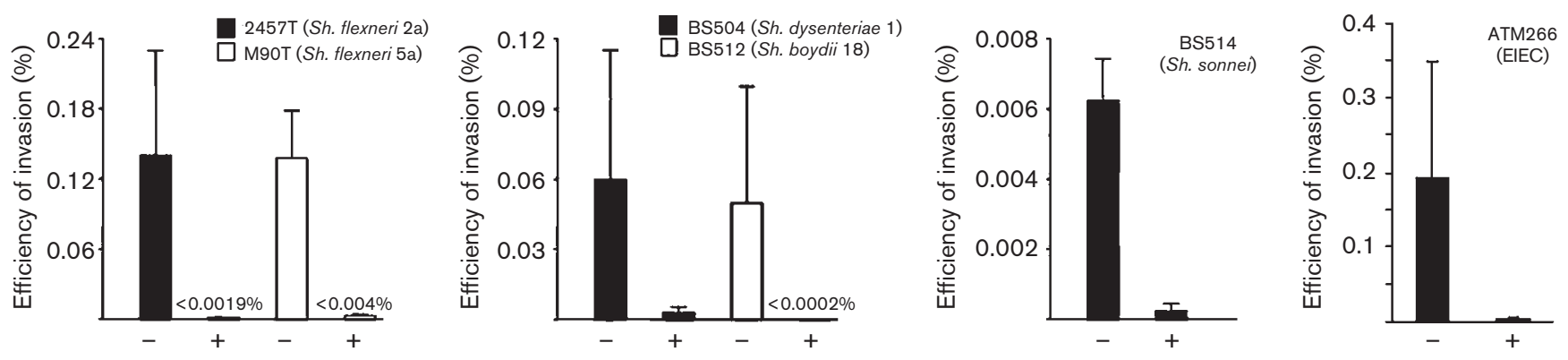

Fig. 5. Effect of QUIN on invasion of mammalian cells by representative strains of Shigella spp. and EIEC. Invasion of L2 cell monolayers (for Shigella) or Hep2 cell monolayers (for EIEC) was measured by the gentamicin protection assay. Hep2 cells were used for EIEC because EIEC are cytotoxic to L2 cells. Percentage invasion was calculated as described in Methods. The results represent the mean \pm SD of data obtained from two to four independent experiments; $(+)$ and $(-)$ indicate the presence or absence of $400 \mu \mathrm{M}$ QUIN during the infection. The data are shown in separate panels with different scales on the $y$-axes due to the different levels of invasion efficiency observed among the species of Shigella and EIEC. 
ium was chosen since, like Shigella, it invades mammalian cells in tissue culture and induces a strong PMN transepithelial migration response upon invasion of T84 cells. Whereas Sh. flexneri 5a invasion of T84 cells was reduced by $73 \%$ with as little as $100 \mu \mathrm{M}$ QUIN (Table 2), the same concentration of QUIN had no effect on invasion of T84 cells by Sal. typhimurium strain $\chi 3306$ (Table 2).

While Shigella induction of PMN transepithelial migration was significantly inhibited by as little as $5 \mu \mathrm{M}$ QUIN (Fig. 4), Sal. typhimurium still induced PMN migration in the presence of $100 \mu \mathrm{M}$ QUIN (Table 2). We also tested E2348/69, an EPEC strain that induces PMN transepithelial migration in this model system. It was still able to induce PMN transepithelial migration in the presence of $100 \mu \mathrm{M}$ QUIN (Table 2). These data strongly suggest that the antivirulence properties of QUIN observed in this assay are limited to Shigella spp.

\section{Effect of QUIN on intracellular growth and virulence factor expression in Shigella}

One possible explanation for the observations that QUIN inhibited cell-to-cell spread and plaque formation, yet did not inhibit intracellular motility of the bacteria is that QUIN inhibits intracellular growth or survival of Shigella. However, when we measured the effect of QUIN on growth of Shigella after invasion of HeLa cells, we found that intracellular growth of the bacteria was not inhibited by $400 \mu \mathrm{M}$ QUIN (data not shown).

Since Shigella can utilize QUIN for growth on minimal medium (data not shown) by converting QUIN to nicotinic acid mononucleotide by the action of the nadC gene product (Fig. 1), we can assume that QUIN can enter the bacterial cell. Therefore, we wished to determine whether the antivirulence effects result from direct repression of Shigella virulence gene expression by QUIN. We used Western blot analysis of the IpaB and IpaC virulence proteins expressed during exponential culture of Sh. flexneri M90T with or without QUIN to address this question. The amount of protein detected in the pellet (non-secreted proteins) plus the amount of protein TCAprecipitated from the supernatant (secreted proteins) were compared for the two conditions. We found less than a twofold reduction in the amount of $\mathrm{IpaB}$ and $\mathrm{IpaC}$ expressed in the presence of QUIN compared to the amount expressed during growth without QUIN $(1.68 \pm 0.12$ - and $1.55 \pm 1.03$-fold reduction for $\mathrm{IpaB}$ and IpaC, respectively). By comparison with the well-studied growth temperature regulation of Shigella virulence, low growth temperature $\left(30^{\circ} \mathrm{C}\right)$ causes 60 -fold reduced expression of ipaB as compared to expression at $37{ }^{\circ} \mathrm{C}$ (Andrews et al., 1991; Hromockyj \& Maurelli, 1989; McCormick et al., 1998). Therefore, it is not likely that the inhibitory effect of QUIN on Shigella virulence is due to QUIN interaction at the level of virulence gene transcription/translation.

\section{Effect of QUIN on secretion of invasion effectors via the Shigella type III secretion system}

Invasion, intracellular and intercellular spread, and the ability to induce PMN transepithelial migration all require the secretion of Ipa proteins from Shigella. Mutations in the T3SS, which mediates secretion of these invasion effectors, abolish these virulence phenotypes (McCormick et al., 1998; Menard et al., 1993; Schuch et al., 1999). We therefore used Western blot analysis of M90T culture supernatants to determine whether QUIN could alter the secretion of Ipa proteins via the T3SS. When wild-type Sh. flexneri are incubated with CR, secretion of Ipa proteins through the T3SS is induced (Parsot et al., 1995). Fig. 6 shows that incubation of M90T with $400 \mu \mathrm{M}$ QUIN strongly impaired the secretion of IpaB and $C$ that is normally observed when bacteria are incubated with CR. Quantitative analysis showed that the secretion of IpaB and $\mathrm{C}$ in the presence of QUIN was reduced $81.9 \%$ and $82.8 \%$, respectively.

We next wanted to examine the specificity of these inhibitory properties of QUIN on the induced secretion of virulence factors. $Y$. enterocolitica was chosen since it is also an enteric pathogen with well-studied T3SS induction by calcium deprivation (Straley et al., 1993). Coomassie staining of SDS-PAGE-analysed protein extracts obtained from Yersinia cultures showed that addition of QUIN to the growth medium did not affect the amount of virulence effectors detected in the supernatant of a calcium-deprived culture (data not shown), which suggests that the effects of QUIN on T3SS-induction are specific to Shigella spp.

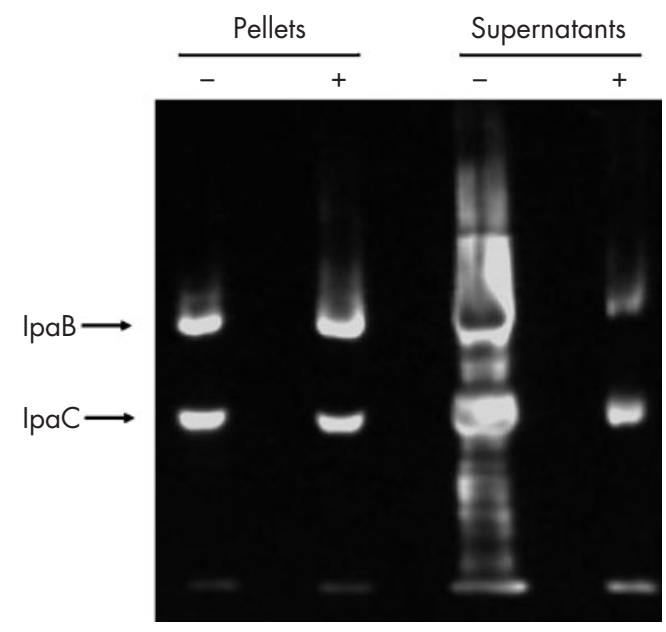

Fig. 6. Effect of QUIN on CR-induced secretion of IpaB and IpaC. Protein extracts were obtained as described in Methods. Identical volumes of pellet and supernatant extracts were subjected to SDS-PAGE and immunoblotted with antibodies directed against $\mathrm{IpaB}$ and IpaC. $(+)$ and $(-)$ indicate the presence or absence of $400 \mu \mathrm{M}$ QUIN during induction. 


\section{nadA $^{+}$nadB $^{+}$transformants of Sh. flexneri 5a are attenuated in virulence}

We wanted to study the effect of reconstructing the NAD pathway in Shigella. The wild-type (functional) nadA and $n a d B$ genes with their native promoters from $E$. coli $\mathrm{K}-12$ were cloned together in pBluescript and transformed into Sh. flexneri 5a strain M90T in order to determine whether expression of these putative AVL in the wild-type Shigella would attenuate virulence. The $n a d A^{+} n a d B^{+}$transformant of M90T, BS813, was able to grow on minimal medium in the absence of nicotinic acid, confirming the functional activity of the transformed genes. Invasion assays with BS813 in HeLa cells showed a 100-fold reduction of invasion capacity compared to M90T $(0.0023 \pm 0.0003 \%$ vs $0.21 \pm 0.03 \%$, respectively). In fact, BS813 gave results similar to BS176, the non-invasive plasmid-cured derivative of M90T (i.e. $0.0019 \pm 0.0001 \%$ ), and to M90T in the presence of $0.5 \mathrm{mM}$ QUIN (Table 1). BS813 was also unable to form plaques in the plaque assay on HeLa cells, even at very high input concentrations (plaque-forming efficiency $<10^{-8}$ ). PMN transmigration experiments could not be performed with BS813 due to the assay's dependence on efficient invasion of Shigella into T84 cells. However, invasion and plaque assay results are sufficient to confirm the antivirulence nature of nadA and $n a d B$ when functional alleles of these genes are expressed in Shigella.

\section{DISCUSSION}

The striking difference between Shigella and E. coli in their ability to synthesize NAD was our rationale for examining the genes in this pathway in Shigella for AVL properties. Shigella is a nicotinic acid auxotroph in that the bacterium is unable to synthesize NAD via the de novo pathway but can still synthesize NAD from exogenous nicotinic acid (Fig. 1). Mutations in $n a d A$ and $n a d B$ are responsible for this auxotrophy in Sh. flexneri 2a (Gemski et al., 1971) and our analysis of these loci in other strains of Shigella and EIEC strongly suggested them as good AVL candidates for Shigella (A.-L. Prunier and others, unpublished).

Several intermediates of the NAD synthesis pathway were tested for their capacity to interfere with the invasive phenotype of Shigella and only QUIN was shown to have an effect. QUIN inhibited three essential phenotypes of Shigella virulence: bacterial invasion, cell-to-cell spread and induction of PMN transepithelial migration. Moreover, we found that QUIN strongly inhibited CR-induced T3SS secretion of the Shigella virulence factors IpaB and IpaC, which are key to the entry of the bacteria into eukaryotic cells and provide a pore for the delivery of other effectors (Enninga et al., 2005; Mounier et al., 1997).

Interference with Shigella-induced actin polymerization could explain how QUIN blocks the entry step. However, any interference of this type must be limited to the early invasion stage, as we observed no gross alteration of the bacteria's ability to polymerize actin in the cytoplasm of the host cell after invasion. When bacteria were allowed to invade cells before addition of QUIN, they produced normal numbers and lengths of cytoplasmic protrusions that contained bacteria. This observation demonstrated the ability of intracellular bacteria to polymerize actin normally in the presence of QUIN.

The plaque assay showed that QUIN also blocked a late stage in Shigella infection, cell-to-cell spread. Since intracellular bacteria were motile in the presence of QUIN and appeared capable of producing cytoplasmic extensions that could 'invade' adjacent cells, the inhibitory effect of QUIN on cell-to-cell spread is not on actin-based motility per se but on some subsequent step in the spreading process. QUIN could be blocking the actual 'invasion' of the bacteria-containing protrusion into the adjacent cell. Alternatively, QUIN could be blocking the ability of the bacteria to lyse the protrusion and escape into the cytoplasm of the adjacent cell.

The failure to spread to adjacent cells is consistent with the inhibition of another virulence phenotype by QUIN, the induction of PMN transepithelial migration. Previous observations in our laboratory have shown that an ics $A$ mutant of Sh. flexneri is severely impaired in its ability to induce transepithelial migration of PMNs (Fernandez et al., 2001). This mutant is unable to catalyse polymerization of actin in the host cytoplasm and, as such, is unable to spread intercellularly. Therefore, induction of PMN transepithelial migration is dependent not only on invasive ability and expression of a functional T3SS (McCormick et al., 1999; Zurawski et al., 2006), but also on the ability to spread to adjacent cells.

While QUIN displays a remarkable ability to inhibit invasion of L2 cells by all four species of Shigella (Fig. 5), its inhibitory properties appear to be restricted to Shigella spp. Sal. typhimurium is also an invasive pathogen of the intestinal epithelium and is closely related to E. coli and Shigella. Both Shigella and Sal. typhimurium invade a variety of cell types in tissue culture, while their respective in vivo targets are different, and they both require the activities of T3SS secretory pathways. Yet QUIN dramatically inhibits Shigella invasion of tissue culture cells whereas it has no effect on invasion by Sal. typhimurium.

Analogous results were observed in the PMN transepithelial migration assay where QUIN blocked PMN signalling by Shigella but had no effect on signalling by Sal. typhimurium. Similarly, a strain of EPEC was insensitive to QUIN and induced PMN transepithelial migration in the presence of $100 \mu \mathrm{M}$ QUIN. It should be noted that, unlike Shigella, Sal. typhimurium has a completely functional pathway for de novo NAD synthesis and synthesizes QUIN (Penfound \& Foster, 1996). The EPEC strain used in this study is a nicotinic acid prototroph and, presumably, also has a functional pathway for de novo NAD synthesis. The model of pathoadaptive evolution by inactivation of AVL predicts that since these pathogens can synthesize 
QUIN, virulence of Sal. typhimurium and EPEC should be insensitive to inhibition by QUIN. Our results confirm this prediction.

The precise mechanism by which QUIN acts to inhibit Shigella virulence is still unknown. It is possible that QUIN acts directly on the mammalian cell to inhibit Shigella invasion. However, pretreatment of HeLa cell monolayers with $0.4 \mathrm{mM}$ QUIN for up to $18 \mathrm{~h}$ prior to infection with M90T had no effect on bacterial invasion of the monolayer cells (data not shown). In addition, pretreatment of the bacteria with QUIN before addition to the cell monolayer was ineffective at blocking bacterial invasion. In fact, inhibition of invasion was only observed when QUIN was present during incubation of the bacteria with the cell monolayer. QUIN did not affect growth of Sh. flexneri in HeLa cells, and it did not significantly impair the expression of IpaB and IpaC virulence factors. It is possible that a bacterial or host target for the action of QUIN only becomes exposed once the bacterium comes in contact with the eukaryotic cell and is subsequently inhibited by QUIN.

QUIN shows a strong inhibitory effect on the induction of T3SS by CR, as shown by reduced levels of secreted IpaB and IpaC when the compound is added during the secretion assay (Fig. 6). The fact that all the Shigella virulence properties monitored in this study (invasion, cell-to-cell spread, and induction of PMN migration) are inhibited by QUIN is consistent with this last observation, since all of these phenotypes require a functional T3SS. As the mediator of T3SS induction by CR has yet to be identified in Shigella, we cannot deduce from this observation the precise mechanism by which QUIN exercises its effect. However, this result is consistent with the hypothesis that a bacterial target for the action of QUIN (probably a T3SS component) only becomes exposed once the virulence properties of the bacterium are induced, either by growth at $37^{\circ} \mathrm{C}$ and addition of CR, or by contact with the eukaryotic cell. Furthermore, the QUIN target is specific to Shigella, since we saw no evidence in this work for the impairment of T3SS function in EPEC, Sal. typhimurium or Y. enterocolitica.

We have proposed two main criteria for the identification of AVL in pathogens. First, the AVL must be present and expressed in closely related or ancestral species occupying the non-virulent ancestral niche but absent from (or mutated in) pathogenic clones living in host tissues. Second, expression of the AVL by the pathogen in host tissues must attenuate virulence (Maurelli, 2007). Therefore, in order to prove that $n a d A / B$ fulfil the definition of AVL, we restored the functionality of nadA/ $B$ in Shigella. We observed a consistent inability of the resulting strain, BS813, to invade tissue culture cells. These results, combined with the results of virulence assays that exhibit a strong inhibitory effect of QUIN on Shigella virulence, demonstrate powerful pathoadaptive selection against the expression of the nadA and $\operatorname{nadB}$ genes in Shigella spp. and show that they are AVL for Shigella.
In summary, we have identified a new set of AVL for Shigella spp., and our results support the concept of bacterial pathogen evolution via inactivation of AVL. The results also demonstrate the potential of genomic and phenotypic screening for differences between bacterial pathogens and related commensals as a powerful tool for discovery of AVL. Nordfelth et al. (2005) recently screened a 9400 compound library to identify small molecules that specifically inhibited the T3SS of Y. pseudotuberculosis. In our study, we used a totally different approach and also identified a small molecule that specifically blocks T3SS of Shigella. Thus, identification of AVL can provide a powerful tool to discover new compounds that specifically inhibit bacterial virulence and therefore could be used in the treatment of infectious diseases. Recognition of pathoadaptive mutation as a process of bacterial pathogen evolution and application of the sort of converse comparative genomics we describe in this report (i.e. identification of genes and/or phenotypes present in a nonpathogenic ancestor and absent in the bacterial pathogen) should enable investigators to identify AVL in other pathogens. This information will provide new avenues for investigation of areas ranging from pathogenic mechanisms to the identification of novel antimicrobial agents.

\section{ACKNOWLEDGEMENTS}

This work was supported by grants AI24656 and AI061058 from the National Institute of Allergy and Infectious Diseases to A.T.M. and grants DK56754 and DK33506 from the National Institute of Diabetes and Digestive and Kidney Diseases to B. A. M. K. L. M. was supported by a T32 training grant sponsored by Harvard Medical School and the Division of Nuclear Medicine at Massachusetts General Hospital. We thank Nancy Strockbine of the CDC for strains of Sh. boydii and Sh. dysenteriae, Gail Hecht of University of Illinois at Chicago for the strain of EPEC, and Peter Feng from FDA for the strain of Yersinia enterocolitica. We thank Nancy Adams for technical assistance, Rachel Binet and Christina Clark for helpful discussions and Daniel Zurawski and Scotty Merrell for careful reading of the manuscript. Finally, we thank Sam Formal, who originally suggested that we look at nadA and $n a d B$ as possible AVL. The opinions or assertions contained herein are the private ones of the authors and are not to be construed as official or reflecting the views of the Department of Defense or the Uniformed Services University of the Health Sciences.

\section{REFERENCES}

Ahmed, Z. U., Sarker, M. R. \& Sack, D. A. (1988). Nutritional requirements of Shigellae for growth in a minimal medium. Infect Immun 56, 1007-1009.

Andrews, G. P., Hromockyj, A. E., Coker, C. \& Maurelli, A. T. (1991). Two novel virulence loci, $m x i A$ and $m x i B$, in Shigella flexneri $2 \mathrm{a}$ facilitate excretion of invasion plasmid antigens. Infect Immun 59, 1997-2005.

Bahrani, F. K., Sansonetti, P. J. \& Parsot, C. (1997). Secretion of Ipa proteins by Shigella flexneri: inducer molecules and kinetics of activation. Infect Immun 65, 4005-4010.

Bernardini, M. L., Mounier, J., d'Hauteville, H., Coquis-Rondon, M. \& Sansonetti, P. J. (1989). Identification of icsA, a plasmid locus of Shigella flexneri that governs bacterial intra- and intercellular spread 
through interaction with F-actin. Proc Natl Acad Sci U S A 86, 3867-3871.

Day, W. A., Jr, Fernandez, R. E. \& Maurelli, A. T. (2001). Pathoadaptive mutations that enhance virulence: genetic organization of the cadA regions of Shigella spp. Infect Immun 69, 7471-7480.

Enninga, J., Mounier, J., Sansonetti, P. \& Tran Van, N. G. (2005). Secretion of type III effectors into host cells in real time. Nat Methods 2, 959-965.

Fasano, A., Noriega, F. R., Liao, F. M., Wang, W. \& Levine, M. M. (1997). Effect of Shigella enterotoxin 1 (ShET1) on rabbit intestine in vitro and in vivo. Gut 40, 505-511.

Fernandez, I. M., Silva, M., Schuch, R., Walker, W. A., Siber, A. M., Maurelli, A. T. \& McCormick, B. A. (2001). Cadaverine prevents the escape of Shigella flexneri from the phagolysosome: a connection between bacterial dissemination and neutrophil transepithelial signaling. J Infect Dis 184, 743-753.

Flachmann, R., Kunz, N., Seifert, J., Gutlich, M., Wientjes, F. J., Laufer, A. \& Gassen, H. G. (1988). Molecular biology of pyridine nucleotide biosynthesis in Escherichia coli. Cloning and characterization of quinolinate synthesis genes nadA and nadB. Eur J Biochem 175, 221-228.

Formal, S. B., Dammin, G. J., Labrec, E. H. \& Schneider, H. (1958). Experimental Shigella infections: characteristics of a fatal infection produced in guinea pigs. J Bacteriol 75, 604-610.

Gemski, P., Formal, S. B. \& Baron, L. S. (1971). Identification of two widely separated loci conferring nicotinic acid dependence on wildtype Shigella flexneri 2a. Infect Immun 3, 500-503.

Groisman, E. A. \& Ochman, H. (1996). Pathogenicity islands: bacterial evolution in quantum leaps. Cell 87, 791-794.

Gulig, P. A. \& Curtiss, R., III (1987). Plasmid-associated virulence of Salmonella typhimurium. Infect Immun 55, 2891-2901.

Harris, J. R., Wachsmuth, I. K., Davis, B. R. \& Cohen, M. L. (1982). High-molecular-weight plasmid correlates with Escherichia coli enteroinvasiveness. Infect Immun 37, 1295-1298.

Hromockyj, A. E. \& Maurelli, A. T. (1989). Identification of Shigella invasion genes by isolation of temperature-regulated inv : lac $Z$ operon fusions. Infect Immun 57, 2963-2970.

Laemmli, U. K. (1970). Cleavage of structural proteins during the assembly of the head of bacteriophage T4. Nature 227, 680-685.

Lee, C. A. (1996). Pathogenicity islands and the evolution of bacterial pathogens. Infect Agents Dis 5, 1-7.

Mantis, N. J. \& Sansonetti, P. J. (1996). The nadB gene of Salmonella typhimurium complements the nicotinic acid auxotrophy of Shigella flexneri. Mol Gen Genet 252, 626-629.

Maurelli, A. T. (2007). Black holes, antivirulence genes, and gene inactivation in the evolution of bacterial pathogens. FEMS Microbiol Lett 267, 1-8.

Maurelli, A. T., Blackmon, B. \& Curtiss, R., III (1984). Loss of pigmentation in Shigella flexneri 2a is correlated with loss of virulence and virulence-associated plasmid. Infect Immun 43, 397-401.

Maurelli, A. T., Fernandez, R. E., Bloch, C. A., Rode, C. K. \& Fasano, A. (1998). 'Black holes' and bacterial pathogenicity: a large genomic deletion that enhances the virulence of Shigella spp. and enteroinvasive Escherichia coli. Proc Natl Acad Sci U S A 95, 3943-3948.

McCormick, B. A., Siber, A. M. \& Maurelli, A. T. (1998). Requirement of the Shigella flexneri virulence plasmid in the ability to induce trafficking of neutrophils across polarized monolayers of the intestinal epithelium. Infect Immun 66, 4237-4243.

McCormick, B. A., Fernandez, M. I., Siber, A. M. \& Maurelli, A. T. (1999). Inhibition of Shigella flexneri-induced transepithelial migration of polymorphonuclear leucocytes by cadaverine. Cell Microbiol 1, $143-155$.
Menard, R., Sansonetti, P. J. \& Parsot, C. (1993). Nonpolar mutagenesis of the ipa genes defines IpaB, IpaC, and IpaD as effectors of Shigella flexneri entry into epithelial cells. J Bacteriol 175, 5899-5906.

Mills, J. A., Buysse, J. M. \& Oaks, E. V. (1988). Shigella flexneri invasion plasmid antigens B and C: epitope location and characterization with monoclonal antibodies. Infect Immun 56, 2933-2941.

Mounier, J., Bahrani, F. K. \& Sansonetti, P. J. (1997). Secretion of Shigella flexneri Ipa invasins on contact with epithelial cells and subsequent entry of the bacterium into cells are growth stage dependent. Infect Immun 65, 774-782.

Nordfelth, R., Kauppi, A. M., Norberg, H. A., Wolf-Watz, H. \& Elofsson, M. (2005). Small-molecule inhibitors specifically targeting type III secretion. Infect Immun 73, 3104-3114.

Oaks, E. V., Wingfield, M. E. \& Formal, S. B. (1985). Plaque formation by virulent Shigella flexneri. Infect Immun 48, 124-129.

Parkos, C. A., Delp, C., Arnaout, M. A. \& Madara, J. L. (1991). Neutrophil migration across a cultured intestinal epithelium. Dependence on a CD11b/CD18-mediated event and enhanced efficiency in physiological direction. J Clin Invest 88, 1605-1612.

Parsot, C., Menard, R., Gounon, P. \& Sansonetti, P. J. (1995). Enhanced secretion through the Shigella flexneri Mxi-Spa translocon leads to assembly of extracellular proteins into macromolecular structures. Mol Microbiol 16, 291-300.

Penfound, T. \& Foster, J. W. (1996). Biosynthesis and recycling of NAD. In Escherichia coli and Salmonella: Cellular and Molecular Biology, pp. 721-730. Edited by F. C. Neidhart and others. Washington, DC: American Society for Microbiology.

Pupo, G. M., Lan, R. \& Reeves, P. R. (2000). Multiple independent origins of Shigella clones of Escherichia coli and convergent evolution of many of their characteristics. Proc Natl Acad Sci U S A 97, 10567-10572.

Sandlin, R. C., Goldberg, M. B. \& Maurelli, A. T. (1996). Effect of O side-chain length and composition on the virulence of Shigella flexneri 2a. Mol Microbiol 22, 63-73.

Sansonetti, P. J., Kopecko, D. J. \& Formal, S. B. (1982). Involvement of a plasmid in the invasive ability of Shigella flexneri. Infect Immun 35, 852-860.

Sansonetti, P. J., Ryter, A., Clerc, P., Maurelli, A. T. \& Mounier, J. (1986). Multiplication of Shigella flexneri within HeLa cells: lysis of the phagocytic vacuole and plasmid-mediated contact hemolysis. Infect Immun 51, 461-469.

Sansonetti, P. J., Tran, V. N. \& Egile, C. (1999). Rupture of the intestinal epithelial barrier and mucosal invasion by Shigella flexneri. Clin Infect Dis 28, 466-475.

Schuch, R., Sandlin, R. C. \& Maurelli, A. T. (1999). A system for identifying post-invasion functions of invasion genes: requirements for the Mxi-Spa type III secretion pathway of Shigella flexneri in intercellular dissemination. Mol Microbiol 34, 675-689.

Sokurenko, E. V., Hasty, D. L. \& Dykhuizen, D. E. (1999). Pathoadaptive mutations: gene loss and variation in bacterial pathogens. Trends Microbiol 7, 191-195.

Straley, S. C., Plano, G. V., Skrzypek, E., Haddix, P. L. \& Fields, K. A. (1993). Regulation by $\mathrm{Ca}^{2+}$ in the Yersinia low-Ca ${ }^{2+}$ response. Mol Microbiol 8, 1005-1010.

Zurawski, D. V., Mitsuhata, C., Mumy, K. L., McCormick, B. A. \& Maurelli, A. T. (2006). OspF and OspC1 are Shigella flexneri type III secretion system effectors that are required for postinvasion aspects of virulence. Infect Immun 74, 5964-5976.

Edited by: I. R. Henderson 